\title{
AN OPTIMAL SCALING LAW FOR FINITE ELEMENT APPROXIMATIONS OF A VARIATIONAL PROBLEM WITH NON-TRIVIAL MICROSTRUCTURE
}

\author{
ANDREW LORENT ${ }^{1}$
}

\begin{abstract}
In this note we give sharp lower bounds for a non-convex functional when minimised over the space of functions that are piecewise affine on a triangular grid and satisfy an affine boundary condition in the second lamination convex hull of the wells of the functional.
\end{abstract}

Mathematics Subject Classification. 74B20, 74S05.

Received: February 26, 2001.

\section{INTRODUCTION}

As shown in the pioneering work of Ball-James [1] and Chipot-Kinderleher [4] the formation of microstructure is closely related to minimising sequences of non-convex functionals for the form:

$$
I(u)=\int_{\Omega} \phi(D u(x)) \mathrm{d} L^{n} x
$$

where $\Omega \subset \mathbb{R}^{n}$ is the reference configuration, $u: \Omega \rightarrow \mathbb{R}^{n}$ is the elastic deformation and $\phi \geq 0$ is the stored energy density which captures the specific material properties. Many features of minimising sequences can be understood by looking at the set $K=\{F: \phi(F)=0\}$ of $\phi$. Due to frame invariance this set is in general of the form $K=S O(n) U_{1} \cup \ldots S O(n) U_{m}$ where symmetric matrices $U_{1}, \ldots U_{m}$ are symmetry related and depend on the symmetry of the phase transition and the transformation strains (see [2] for detailed discussion). The simplest non-trivial problem is called the two well problem; this correspond to the set

$$
K=S O(2) \cup S O(2) H
$$

where $H$ is a diagonal matrix with eigenvalues $0<\mu \leq \lambda$ and $\lambda \mu \geq 1$.

An important question was to determine the set $K^{\text {macro }}$ of macroscopically zero energy states. By definition this consists of all $F \in M^{n \times n}$ for which there exists a sequence of uniformly Lipschitz maps $u_{j}: \Omega \rightarrow \mathbb{R}^{n}$ such that

$$
\begin{gathered}
u_{j}(x)=F x \text { for all } x \in \partial \Omega \\
\int_{\Omega} d\left(D u_{j}(x), K\right) \mathrm{d} L^{n} x \rightarrow 0 \text { as } j \rightarrow \infty .
\end{gathered}
$$

Keywords and phrases. Finite-well non-convex functionals, finite element approximations.

1 Max-Planck-Institute, Inselstr. 22-26, 04103 Leipzig, Germany. e-mail: lorent@mis.mpg.de 
A covering argument shows that $K^{\text {macro }}$ is independent of $\Omega$. In fact it agrees with the so called quasi-convex hull $K^{q c}$ (see [8] for an overview of the relevant notions) and has been computed explicitly for the two well problem in [10]: Given a set of matrices $A$ we can form the first lamination convex hull of $A$ as follows:

$$
A^{(1)}=\left\{\lambda G+(1-\lambda) H: G, H \in K, G-H=a \otimes m, \text { for } a, m \in \mathbb{R}^{n}, \lambda \in[0,1]\right\},
$$

the second lamination convex hull of $A$ (denoted by $A^{(2)}$ ) is just the first lamination convex hull of $A^{(1)}$. It was shown in [10] that in the case $\operatorname{det}(H)=1 ; K^{q c}=K^{(2)}$.

Following work of Luskin and coworkers there has been much interest in numerical minimisation of multi-well problems and in optimal scaling laws for finite element approximations (see [6] for a survey and, [3], [7] for more recent developments). In particular it has been shown that for a quasi-uniform triangulation $\tau_{h}$, letting $A_{h}$ denote the space of functions $u: \Omega \rightarrow \mathbb{R}^{n}$ that are piecewise affine on the triangles of $\tau_{h}$ and satisfy affine boundary condition; $u(x)=F$ for all $x \in \partial \Omega$ where $F \in K^{(2)}$, then

$$
\inf _{u \in A_{h}} \int_{\Omega} d(D u(x), K) \mathrm{d} L^{2} x \leq C h^{\frac{1}{3}} .
$$

This applies in particular to the two well problem. The goal of this paper is to show that the scaling exponent $\frac{1}{3}$ is sharp.

Theorem. Denote $\Omega=[0,1]^{2}$, and let $e_{1}, e_{2}$ be the coordinate axis. Fix $h \in\left(0, \frac{1}{64}\right)$ and let $\tau_{h}$ be a regular triangulation of the plane with mesh size $h$, with the property that none of the mesh triangles has an edge parallel to the vector $e_{1}$. Let

where:

$$
K=\{U, L, R\},
$$

$$
U=\left(\begin{array}{ll}
1 & 0 \\
0 & 0
\end{array}\right), L=\left(\begin{array}{cc}
-1 & 0 \\
0 & -1
\end{array}\right), R=\left(\begin{array}{cc}
-1 & 0 \\
0 & 1
\end{array}\right) .
$$

Finally, let $A_{h}$ denote the set of functions $f: \Omega \rightarrow \mathbb{R}^{2}$ that vanish on the boundary of $\Omega$, and are Lipschitz continuous and affine on each triangle of $\tau_{h}$ intersecting $\Omega$, then

$$
\inf _{u \in A_{h}} \int_{\Omega} d(D u(x), K) \mathrm{d} L^{2} x \geq c h^{1 / 3},
$$

with some some constant $c>0$ depending only on the choice of the triangulation $\tau_{h}$.

In view of possible applications we make an attempt to keep the constant $c$ within reasonable numerical bounds. For a specific choice of grid, our reasoning will be carried out for

$$
c>\frac{1}{500000} \text {. }
$$

The article is organized as follows. Section 2 contains the proof of our theorem, for the convenience of the reader, we have divided it into several steps. In Section 3 we discuss a potential application of results of our type to the problem of determining the quasi-convex hull of a three well problem in three dimensions related to the cubic to tetragonal transition.

\section{Proof of The Theorem}

Strategy of proof. Figure 1 shows a second order laminate having derivatives mostly in the wells $K$ and vanishing on the boundary, we will refer to this as function $D$. By taking the function in $\widetilde{D} \in A_{h}$ that approximates $D$ (i.e. for every triangle $T$ in $\tau_{h}, \widetilde{D}$ on $T$ is equal to the affine function given by the interpolation of the values of $D$ at the corners of the triangle). Note that for each crease in the function $D$ (i.e. each line 


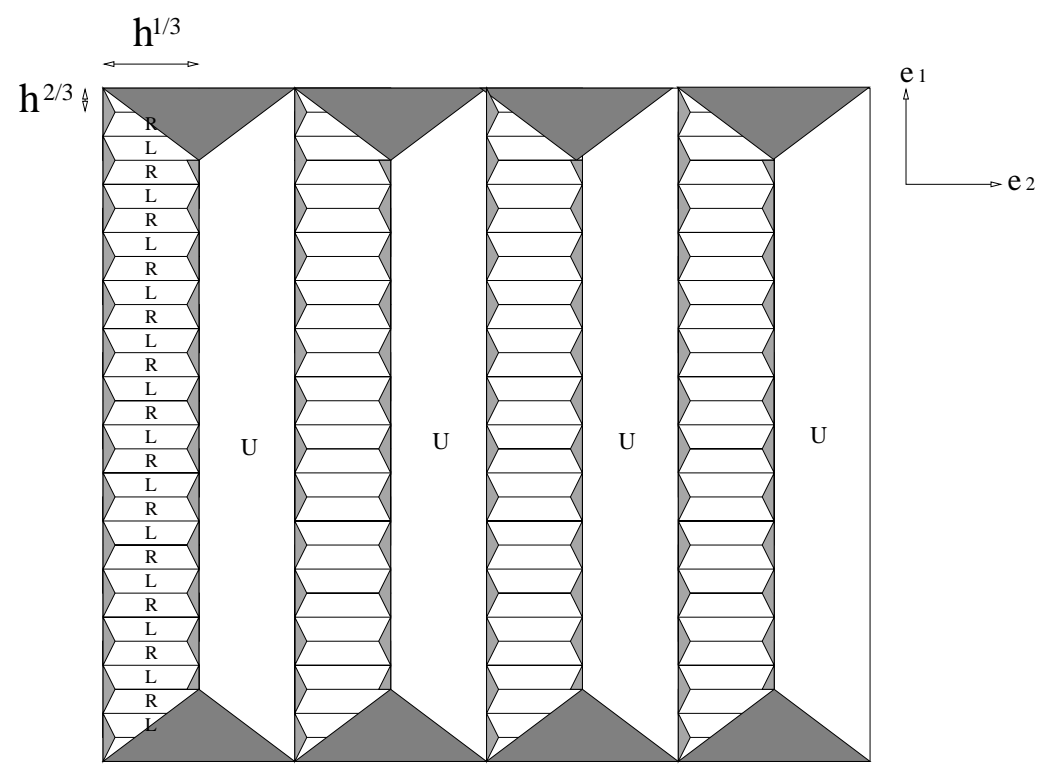

FIGURE 1.

where there is a jump in the derivative of $\mathrm{D}$ ) the function $\widetilde{D}$ will have a line of triangles some distance away from the wells, in this way, our functional is in some sense the sum of a surface energy term and a bulk energy term $^{1}$. With this observation it is possible to see that $\int_{\Omega} d(D u(x), K) \mathrm{d} L^{2} x<C h^{\frac{1}{3}}$. The strategy of the proof is motivated largely by this example.

If we have a function $u \in A_{h}$ with $\int_{\Omega} d(D u(x), K) \mathrm{d} L^{2} x<c h^{\frac{1}{3}}$ then for many triangles we must have that $D u$ is close to the wells $K$, so in particular the derivatives of the coordinate functions $u_{1}=u \cdot e_{1}$ and $u_{1}=u \cdot e_{1}$ are very restricted. Just from the fact that for most triangles $\left|\frac{\partial u_{1}}{\partial x_{2}}\right|$ is small, by integrating from the boundary we have the $L^{1}$ norm of the function $u_{2}$ cannot be too big, this forces the function $u$ to oscillate in some sense like the first laminate of the function $D$. More specifically, we define vertical blocks of squares that are $h^{\frac{1}{3}}$ in width and 1 in height and we show that $\left|u_{2}\right|$ is less that $\frac{h^{\frac{1}{3}}}{50}$ for most of these blocks. The next step is to try and show $u$ oscillates in these blocks on a scale of roughly $h^{\frac{2}{3}}$, i.e. that $u$ oscillates like the second lamination of $D$. This can be done by using carefully the specific properties of the coordinate functions $u_{1}$ and $u_{2}$ on the squares for which $D u$ is close to $K$. We will define minirows in our blocks which are just horizontal rows of $\left[\frac{1}{h^{\frac{2}{3}}}\right]$ squares and by simple counting arguments we will obtain a set of at least $c_{1}\left[\frac{1}{h^{\frac{2}{3}}}\right]$ minirows all spaced out from each other by at least $\left[\frac{1}{h^{\frac{2}{3}}}\right]$ squares with the property that on each minirow the function $u_{1}$ has a set $A$ of at least $c_{2}\left[\frac{1}{h^{\frac{2}{3}}}\right]$ squares for which $\frac{\partial u_{1}}{\partial x_{1}} \approx 1$ and a set $B$ of at least $c_{2}\left[\frac{1}{h^{\frac{2}{3}}}\right]$ squares for which $\frac{\partial u_{1}}{\partial x_{1}} \approx-1$. Now we consider the rectangle of squares which is $\left[\frac{1}{h^{\frac{1}{3}}}\right]$ squares in height whose base is the minirow. Either for half the squares $\theta \in A$, the line of squares in the rectangle above $\theta$ have $\frac{\partial u_{1}}{\partial x_{1}} \approx 1$ or for half there is some square in the line for which $u$ has a change of derivative. Since a change in derivative forces there to be at least one triangle on which $D u$ is not close to $K$, the latter possibility gives us $\frac{c_{2}}{2}\left[\frac{1}{h^{\frac{2}{3}}}\right]$ such triangles. Now as we have proportionally the same amount or error inside a rectangle of the same size in the domain of function $\widetilde{D}$, too many rectangles of this type will give us the lower bound. On the other hand if this does not happen for the set $A$ and the set $B$ then since $\frac{\partial u_{1}}{\partial x_{1}} \approx 1 \Longrightarrow \frac{\partial u_{2}}{\partial x_{2}} \approx 0$ and $\frac{\partial u_{1}}{\partial x_{1}} \approx-1 \Longrightarrow \frac{\partial u_{2}}{\partial x_{2}} \approx 1$ or $\frac{\partial u_{2}}{\partial x_{2}} \approx-1$, coupled with the

\footnotetext{
${ }^{1}$ I would like to thank G. Dolzmann for pointing this out to me.
} 
fact that $\frac{\partial u_{2}}{\partial x_{1}} \approx 0$ for all triangles with $D u$ close to $K$, integrating in the $e_{2}$ direction inside our rectangle gives that the error inside the rectangle is at least $c_{3} h^{\frac{4}{3}}$. So in either case adding up the error inside each rectangle gives us the lower bound, this is the strategy of the proof.

Step 1. To simplify the arguments, we prove our result for a specific choice of the triangulation $\tau_{h}$, it is not difficult to see that the same reasoning could be carried out for any other mesh that satisfies the hypothesis of the theorem.

Denote $S_{i, j}:=\left[(j-1) h e_{1}, j h e_{1}\right] \otimes\left[(i-1) h e_{2}, i h e_{2}\right]$ the $(i, j)$-th square in $\Omega$, and let $D_{i, j}$ be the equilateral diamond inside $S_{i, j}$ whose corners touch the midpoints of the sides of the square. If we cut $D_{i, j}$ vertically down the center we get two triangles, the left hand one denoted by $T_{i, j}^{(1)}$ and the right hand one by $T_{i, j}^{(2)}$; let now $T_{i, j}^{(3)}$ be the triangle obtained as the union of the bottom left triangle of $S_{i, j} \backslash D_{i, j}$ and the top left triangle of $S_{i-1, j} \backslash D_{i-1, j}$. Finally, $T_{i, j}^{(4)}$ stands for the triangle obtained as the union of the bottom right triangle of $S_{i, j} \backslash D_{i, j}$ and the top right triangle of $S_{i-1, j} \backslash D_{i-1, j}$. Our triangulation $\tau_{h}$ is given by

$$
\tau_{h}:=\left\{T_{i, j}^{(k)}: k \in\{1,2,3,4\}, i, j \in \mathbb{N}\right\} .
$$

Suppose the result was not true and thus we could find grid size $h$ and a function $u \in A_{h}$ with the property that

$$
\int_{\Omega} d(D u(x), K) \mathrm{d} L^{2} x<c h^{1 / 3}
$$

for some $c<\frac{1}{500000}$. Define the set of "bad squares"

$$
B=\left\{S_{i, j}: \int_{S_{i, j}} d(D u(x), K) \mathrm{d} L^{2} x>\frac{h^{2}}{100}\right\}
$$

and the set of "good squares"

$$
G=\left\{S_{i, j}: S_{i, j} \notin B\right\}
$$

Note that $\operatorname{card}(B) \frac{h^{2}}{100} \leq \frac{h^{\frac{1}{3}}}{500000}$, so consequently

$$
\operatorname{card}(B)<\frac{h^{\frac{1}{3}}}{5000 h^{2}}=\frac{1}{5000 h^{\frac{5}{3}}} .
$$

Let $N=\left[h^{-1}\right]$ and let $n_{0}=\left[N h^{\frac{1}{3}}\right]$. We split the set $\Omega$ into $\left[\frac{N}{n_{0}}\right]$ blocks of touching columns

$$
B_{k}=\bigcup_{\substack{i \in\{1, \ldots, N\} \\ j \in\left\{(k-1) n_{0}, \ldots, k n_{0}\right\}}} S_{i, j}, \text { for } k=1,2, \ldots,\left[\frac{N}{n_{0}}\right] .
$$

In the following steps we will obtain information from the properties of the coordinate functions $u_{1}$ and $u_{2}$ of the vector valued function $u$,

$$
u(x)=\left(\begin{array}{l}
u_{1}(x) \\
u_{2}(x)
\end{array}\right)
$$


Step 2. In this step we will use estimate (1) to show that the function $u_{1}$ does not grow "too big" over most of the blocks of $\Omega$. Note first that $D u(x) \in K$ implies:

$$
\frac{\partial u_{1}}{\partial x_{1}} \in\{1,-1\} \text { and } \frac{\partial u_{1}}{\partial x_{2}}=0
$$

Let $\gamma:[0, N h] \rightarrow \mathbb{R}$ be defined by

$$
\gamma(x)=\sup \left\{\left|u_{1}(z)\right|: z \in\left\{x e_{1}+\left\langle e_{2}\right\rangle\right\} \cap \Omega\right\},
$$

and let $P_{1}$ stand for the projection of $\mathbb{R}^{2}$ onto the $\left\langle e_{1}\right\rangle$ axis.

We are going to show that for most blocks $B_{k}$, the average of $\gamma$ over $P_{1}\left(B_{k}\right)$ is "not too big". To make this more precise, define

$$
T=\left\{k \in\left\{1,2, \ldots,\left[\frac{N}{n_{0}}\right]\right\}: \int_{P_{1}\left(B_{k}\right)} \gamma(x) \mathrm{d} L^{1} x>\frac{h^{\frac{2}{3}}}{50000}\right\} .
$$

For any fixed $k \in T$ and any $t \in P_{1}\left(B_{k}\right)$ we can find $z_{t} \in\left\{t e_{1}+\left\langle e_{2}\right\rangle\right\} \cap \Omega$ such that $\left|u_{1}(z)\right|=\gamma(t)$ and hence

$$
\begin{aligned}
\gamma(t) & =\left|u_{1}(z)\right|=\left|\int_{0}^{z} \frac{\partial u_{1}}{\partial x_{2}}(x) \mathrm{d} L^{1} x\right| \leq \int_{0}^{z}\left|\frac{\partial u_{1}}{\partial x_{2}}(x)\right| \mathrm{d} L^{1} x \\
& \leq \int_{P_{1}^{-1}(t) \cap \Omega} d(D u(x), K) \mathrm{d} L^{1} x .
\end{aligned}
$$

Using the Fubini theorem,

$$
\int_{P_{1}\left(B_{k}\right)} \gamma(y) \mathrm{d} L^{1} y \leq \int_{\Omega \cap P_{1}^{-1}\left(P_{1}\left(B_{k}\right)\right)} d(D u(x), K) \mathrm{d} L^{2} x
$$

As the sets $\Omega \cap P_{1}^{-1}\left(P_{1}\left(B_{k}\right)\right)$ are pairwise disjoint we have that

$$
\begin{aligned}
\operatorname{card}(T) \cdot \frac{h^{\frac{2}{3}}}{50000} & \leq \int_{\Omega} d(D u(x), K) \mathrm{d} L^{2} x \\
& \leq \frac{h^{\frac{1}{3}}}{500000}
\end{aligned}
$$

and consequently

$$
\operatorname{card}(T) \leq \frac{1}{10 h^{\frac{1}{3}}},
$$

as claimed.

Define

Note that

$$
\Phi_{1}=\left\{n \in\left\{1,2, \ldots,\left[\frac{N}{n_{0}}\right]\right\}: n \notin T\right\} .
$$

$$
\operatorname{card}\left(\Phi_{1}\right) \geq \frac{N}{n_{0}}-\frac{1}{10 h^{\frac{1}{3}}} \geq \frac{9}{10 h^{\frac{1}{3}}} .
$$

From this point on we work inside the blocks of the set $\Phi_{1}$; refining onto regions of $\Omega$ that are well controlled will be a continuing theme. 
Step 3. Now we refine the minirows inside the blocks indexed by $\Phi_{1}$. Let

$$
R_{k, i}=\left\{S_{k, j}: j \in\left\{(i-1) n_{0}, \ldots, i n_{0}\right\}\right\}
$$

be the $k$-th minirow in the $i$-th block. Now for any $i \in \Phi_{1}$, let

$$
O_{i}=\left\{k \in\{1, \ldots N\}: \operatorname{card}\left(B \cap R_{k, i}\right) \geq \frac{n_{0}}{500}\right\} .
$$

Since by $(2), \operatorname{card}\left(O_{i}\right) \cdot \frac{n_{0}}{500} \leq \frac{1}{5000 h^{\frac{5}{3}}}$, then

$$
\operatorname{card}\left(O_{i}\right) \leq \frac{1}{10 n_{0} h^{\frac{5}{3}}} .
$$

Together with $n_{0}>h^{-\frac{2}{3}}-2$, this yields

$$
\operatorname{card}\left(O_{i}\right) \leq \frac{1}{10\left(h-2 h^{\frac{5}{3}}\right)} \leq \frac{1}{\frac{30}{4} h}=\frac{4}{30 h} .
$$

Denote

$$
\begin{aligned}
M_{i} & =\{1,2, \ldots N\} \backslash O_{i}, \\
Q_{i} & =\left\{k \in M_{i}: \sup \left\{d(D u(x), K): x \in R_{k, i}\right\}<\frac{1}{5000 h^{\frac{2}{3}}}\right\} .
\end{aligned}
$$

Note that, $\operatorname{card}\left(M_{i}\right) \geq \frac{26}{30 h}$.

For each $k \in Q_{i}$, since $u$ is piecewise affine, there exists at least one triangle on which the derivative of $u$ is at least $\frac{1}{5000 h^{\frac{2}{3}}}$ away from the wells $K$. Thus

$$
\int_{R_{k, i}} d(D u(x), K) \mathrm{d} L^{2} x>\frac{h^{2}}{40000 h^{\frac{2}{3}}}
$$

and

$$
\operatorname{card}\left(Q_{i}\right) \frac{h^{2}}{40000 h^{\frac{2}{3}}}<\frac{h^{\frac{1}{3}}}{500000},
$$

as well as card $\left(Q_{i}\right)<\frac{h^{\frac{1}{3}}}{10 h^{\frac{4}{3}}}=\frac{1}{10 h}$. Finally, denoting $N_{i}=M_{i} \backslash Q_{i}$, we get

$$
\operatorname{card}\left(N_{i}\right) \geq \frac{26}{30 h}-\frac{1}{10 h}=\frac{23}{30 h} .
$$

Introduce another refinement to the minirows inside the block $B_{i}$

$$
J_{i}=\left\{k \in N_{i}: \int_{R_{k, i}} d(D u(z), K) \mathrm{d} L^{2} z<\frac{h^{\frac{4}{3}}}{50000}\right\} .
$$

Since $\operatorname{card}\left(N_{i} \backslash J_{i}\right) \cdot \frac{h^{\frac{4}{3}}}{50000}<\frac{h^{\frac{1}{3}}}{500000}$, then $\operatorname{card}\left(N_{i} \backslash J_{i}\right)<\frac{1}{10 h}$ and thus

$$
\operatorname{card}\left(J_{i}\right) \geq \frac{2}{3 h} .
$$


Now we apply a final refinement of the minirows, to distinguish only those minirows for which $\left|u_{1}\right|$ does not "get too big". For any $i \in \Phi_{1}$ let

$$
H_{i}=\left\{j \in J_{i}: \sup \left\{\left|u_{1}(z)\right|: z \in R_{k, i}\right\} \leq \frac{h^{\frac{1}{3}}}{50}\right\} .
$$

Our claim is that $J_{i} \backslash H_{i}=\emptyset$.

Suppose not and let $i \in \Phi_{1}$ be such that there exists a number $k \in J_{i} \backslash H_{i}$. Take $x \in R_{k, i}$ such that

$$
\left|u_{1}(x)\right|=\sup \left\{\left|u_{1}(z)\right|: z \in R_{k, i}\right\}
$$

Now $x$ belongs so some square $S_{k, j}$ and so for any other point $z \in S_{k, j}$ there holds

$$
u_{1}(z)>u_{1}(x)-\operatorname{Lip}(u)|x-z|>u_{1}(x)-\operatorname{Lip}(u) h .
$$

As $k \in Q_{i}$ so $\operatorname{Lip}(u)<\frac{1}{2500 h^{\frac{2}{3}}}$ and it follows that

$$
u_{1}(z)>\frac{100 h^{\frac{1}{3}}}{5000}-\frac{2 h}{5000 h^{\frac{2}{3}}}=\frac{98 h^{\frac{1}{3}}}{5000} .
$$

Note moreover that for any horizontal line $l$ through our minirow $R_{k, i}$, (that is $l=R_{k, i} \cap\left\{\lambda e_{2}+\left\langle e_{1}\right\rangle\right\}$ for some $\lambda \in \mathbb{R})$ we have that $\sup \left\{|u(z)|: z \in l \cap R_{k, i}\right\}>\frac{98 h^{\frac{1}{3}}}{5000}$.

Let $x \in l$ be such that $|u(x)|>\frac{98 h^{\frac{1}{3}}}{5000}$. Since $i \in \Phi_{1}$,

$$
\int_{l}\left|u_{1}(t)\right| \mathrm{d} L^{1} t x<\frac{h^{\frac{2}{3}}}{50000}
$$

so there exists a point $y \in B_{\frac{h^{\frac{1}{3}}}{200}}(x) \cap l$ for which $u_{1}(y) \leq \frac{h^{\frac{1}{3}}}{200}$. Hence

$$
\begin{aligned}
\frac{73 h^{\frac{1}{3}}}{5000} & \leq\left|u_{1}(y)-u_{1}(x)\right|=\left|\int_{x}^{y} \frac{\partial u_{1}}{\partial x_{1}}(z) \mathrm{d} L^{1} z\right| \\
& \leq \int_{x}^{y}\left|\frac{\partial u_{1}}{\partial x_{1}}(z)\right| \mathrm{d} L^{1} z
\end{aligned}
$$

and

$$
\begin{aligned}
\int_{x}^{y}|| \frac{\partial u_{1}}{\partial x_{1}}(z)|-1| \mathrm{d} L^{1} z & \geq \int_{x}^{y}\left|\frac{\partial u_{1}}{\partial x_{1}}(z)\right|-1 \mathrm{~d} L^{1} z \\
& \geq \frac{73 h^{\frac{1}{3}}}{5000}-|x-y| \geq \frac{73 h^{\frac{1}{3}}}{5000}-\frac{25 h^{\frac{1}{3}}}{5000} \\
& \geq \frac{48 h^{\frac{1}{3}}}{5000}
\end{aligned}
$$

Finally

$$
\begin{aligned}
\int_{l} d(D u(x), K) \mathrm{d} L^{1} x & \geq \int_{x}^{y}|| \frac{\partial u_{1}}{\partial x_{1}}(z)|-1| \mathrm{d} L^{1} z \\
& \geq \frac{48 h^{\frac{1}{3}}}{5000}
\end{aligned}
$$


which is true for every horizontal line through $R_{k, j}$. The Fubini theorem yields now

$$
\int_{R_{k, j}} d(D u(z), K) \mathrm{d} L^{2} z \geq \frac{48 h^{\frac{4}{3}}}{5000}
$$

contradicting the fact that $k \in J_{i}$ and proving our claim.

Step 4. In this step we use a covering theorem to refine the minirows in each block indexed by $\Phi_{1}$ to a subset containing only minirows that are pairwise spaced out from each other by at least $\left[\frac{2}{h^{\frac{1}{3}}}\right]$ squares.

The family of intervals $\left\{\left[j h-h^{\frac{2}{3}}, j h+h^{\frac{2}{3}}\right]: j \in J_{i}\right\}$ forms a covering of the set

$$
A=\bigcup_{j \in J_{i}}[(j-1) h, j h] .
$$

As in view of (4),

$$
L^{1}(A) \geq \frac{2}{3 h} h \geq \frac{2}{3}
$$

the $5 r$ Covering Theorem (see for example [9] Th. 2.1) provides us with a set $V_{i} \subset J_{i}$ with the following two properties:

- $A \subset \bigcup_{j \in V_{i}}\left[j h-5 h^{\frac{2}{3}}, j h+5 h^{\frac{2}{3}}\right]$.

- The intervals $\left[j h-h^{\frac{2}{3}}, j h+h^{\frac{2}{3}}\right], j \in V_{i}$ are pairwise disjoint.

The first point above implies immediately that $10 h^{\frac{2}{3}} \operatorname{card}\left(V_{i}\right) \geq \frac{2}{3}$ and so

$$
\operatorname{card}\left(V_{i}\right) \geq \frac{2}{30 h^{\frac{2}{3}}} \text {. }
$$

Fix a square $S_{k, j} \in G$. Since $u$ is piecewise affine on each $T_{k, j}^{(1)}$ and $T_{k, j}^{(2)}$ and $D u$ on this square stays "close" to the wells so on both sides of the diamond $D_{k, j}$ the gradient $D u$ must be close to the same well, as $e_{2}$ is not a rank one connection for the wells. Thus either

$$
\sup \left\{\left|\frac{\partial u_{1}}{\partial x_{1}}(z)-1\right|: z \in D_{k, j}\right\}<\frac{1}{25} \quad \text { or } \quad \sup \left\{\left|\frac{\partial u_{1}}{\partial x_{1}}(z)+1\right|: z \in D_{k, j}\right\}<\frac{1}{25} .
$$

Denote

$$
\begin{aligned}
& P_{1}=\left\{S_{i, j} \in G: \sup \left\{\left|\frac{\partial u_{1}}{\partial x_{1}}(z)-1\right|: z \in D_{i, j}\right\}<\frac{1}{25}\right\} \\
& D_{1}=\left\{S_{i, j} \in G: \sup \left\{\left|\frac{\partial u_{1}}{\partial x_{1}}(z)+1\right|: z \in D_{i, j}\right\}<\frac{1}{25}\right\} .
\end{aligned}
$$

Take $j \in \Phi_{1}$ and $i \in V_{j}$, we consider the minirow $R_{i, j}$. Let $a_{0}=\left((j-1) n_{0} h,\left(i+\frac{1}{2}\right) h\right)$ be the center point at the left of the minirow. Also, for $k=1,2, \ldots, n_{0}$ set $a_{k}=\left(\left((j-1) n_{0}+k\right) h,\left(i+\frac{1}{2}\right) h\right)$. Define

$$
\begin{aligned}
M & =\left\{k \in\left\{1,2, \ldots, n_{0}\right\}: S_{i,(j-1) n_{0}+k} \in P_{1}\right\}, \\
L & =\left\{k \in\left\{1,2, \ldots, n_{0}\right\}: S_{i,(j-1) n_{0}+k} \in D_{1}\right\}, \\
R & =\left\{1,2, \ldots, n_{0}\right\} \backslash M \cup L .
\end{aligned}
$$


Since $G=P_{1} \cup D_{1}$ we get $\operatorname{card}(R) \leq \frac{n_{0}}{500}$ and

$$
\begin{aligned}
u\left(a_{n_{0}}\right)-u\left(a_{0}\right)= & \sum_{k=1}^{n_{0}} u\left(a_{k}\right)-u\left(a_{k-1}\right)=\sum_{k=1}^{n_{0}} \int_{a_{k-1}}^{a_{k}} \frac{\partial u_{1}}{\partial x_{1}}(z) \mathrm{d} L^{1} z \\
= & \sum_{k \in M} \int_{a_{k-1}}^{a_{k}} \frac{\partial u_{1}}{\partial x_{1}}(z) \mathrm{d} L^{1} z+\sum_{k \in L} \int_{a_{k-1}}^{a_{k}} \frac{\partial u_{1}}{\partial x_{1}}(z) \mathrm{d} L^{1} z \\
& +\sum_{k \in R} \int_{a_{k-1}}^{a_{k}} \frac{\partial u_{1}}{\partial x_{1}}(z) \mathrm{d} L^{1} z \\
\geq & \operatorname{card}(M) h\left(1-\frac{1}{25}\right)-\operatorname{card}(L) h\left(1+\frac{1}{25}\right) \\
& +\sum_{k \in R} \int_{a_{k-1}}^{a_{k}} \frac{\partial u_{1}}{\partial x_{1}}(z) \mathrm{d} L^{1} z .
\end{aligned}
$$

On the other hand by the choice of $k \in H_{i}$ there holds

$$
u\left(a_{n_{0}}\right)-u\left(a_{0}\right) \leq\left|u\left(a_{n_{0}}\right)\right|+\left|u\left(a_{0}\right)\right| \leq \frac{h^{\frac{1}{3}}}{25}
$$

Step 5. For our chosen minirow $R_{i, j}$ we are going to show the following inequality

$$
\operatorname{card}(M) \leq \operatorname{card}(L)+\frac{n_{0}}{5}
$$

Suppose the converse inequality was true. Then

$$
\begin{aligned}
\frac{h^{\frac{1}{3}}}{25} \geq & \left(\operatorname{card}(L)+\frac{n_{0}}{5}\right) h\left(1-\frac{1}{25}\right)-\operatorname{card}(L) h\left(1+\frac{1}{25}\right) \\
& -\sum_{k \in R} \int_{a_{k-1}}^{a_{k}} \frac{\partial u_{1}}{\partial x_{1}}(z) \mathrm{d} L^{1} z \\
= & -\operatorname{card}(L) \frac{2 h}{25}+\frac{n_{0} h}{5}\left(1-\frac{1}{25}\right)+\sum_{k \in R} \int_{a_{k-1}}^{a_{k}} \frac{\partial u_{1}}{\partial x_{1}}(z) \mathrm{d} L^{1} z,
\end{aligned}
$$

and using card $(L) \leq n_{0}$ we receive

$$
\begin{aligned}
\frac{3 h^{\frac{1}{3}}}{25} & \geq \frac{n_{0} h}{5} \frac{24}{25}+\sum_{k \in R} \int_{a_{k-1}}^{a_{k}} \frac{\partial u_{1}}{\partial x_{1}}(z) \mathrm{d} L^{1} z \\
& \geq\left(h^{-\frac{2}{3}}-1\right) h \frac{24}{125}+\sum_{k \in R} \int_{a_{k-1}}^{a_{k}} \frac{\partial u_{1}}{\partial x_{1}}(z) \mathrm{d} L^{1} z \\
& \geq\left(h^{\frac{1}{3}}-h\right) \frac{24}{125}+\sum_{k \in R} \int_{a_{k-1}}^{a_{k}} \frac{\partial u_{1}}{\partial x_{1}}(z) \mathrm{d} L^{1} z \\
& \geq \frac{3}{4} h^{\frac{1}{3}} \frac{24}{125}+\sum_{k \in R} \int_{a_{k-1}}^{a_{k}} \frac{\partial u_{1}}{\partial x_{1}}(z) \mathrm{d} L^{1} z
\end{aligned}
$$


and

$$
\frac{60 h^{\frac{1}{3}}}{500}-\frac{72 h^{\frac{1}{3}}}{500} \geq \sum_{k \in R} \int_{a_{k-1}}^{a_{k}} \frac{\partial u_{1}}{\partial x_{1}}(z) \mathrm{d} L^{1} z
$$

Let $\tilde{R} \subset R$ be the set of these $k \in R$ such that

$$
\int_{a_{k-1}}^{a_{k}} \frac{\partial u_{1}}{\partial x_{1}}(z) \mathrm{d} L^{1} z \leq 0
$$

note that we have $\frac{\partial u_{1}}{\partial x_{1}}(z)<0$ for all $z \in\left[a_{k-1}, a_{k}\right]$ for $k \in R$. Now

$$
\frac{-12 h^{\frac{1}{3}}}{500} \geq \sum_{k \in \tilde{R}} \int_{a_{k-1}}^{a_{k}} \frac{\partial u_{1}}{\partial x_{1}}(z) \mathrm{d} L^{1} z
$$

but

$$
\begin{aligned}
\sum_{k \in \tilde{R}} \int_{a_{k-1}}^{a_{k}}\left|\frac{\partial u_{1}}{\partial x_{1}}(z)+1\right| \mathrm{d} L^{1} z & \geq \sum_{k \in \tilde{R}} \int_{a_{k-1}}^{a_{k}}\left|\frac{\partial u_{1}}{\partial x_{1}}(z)\right|-1 \mathrm{~d} L^{1} z \\
& \geq \frac{12 h^{\frac{1}{3}}}{500}-\operatorname{card}(\tilde{R}) h \geq \frac{11 h^{\frac{1}{3}}}{500} .
\end{aligned}
$$

Now since $u$ is piecewise affine on each triangle $T_{k, j}^{(1)}, T_{k, j}^{(2)}$ then if we let $t_{k, j}^{(1)}$ and $t_{k, j}^{(2)}$ be respectively the centers of $T_{k, j}^{(1)}$ and $T_{k, j}^{(2)}$ we obtain

$$
\begin{aligned}
\frac{\partial u_{1}}{\partial x_{1}}\left(t_{k, j}^{(i)}\right) & =\left.\frac{\partial u_{1}}{\partial x_{1}}\right|_{T_{k, j}^{(i)}} \text { for } i=1,2 \\
\int_{a_{k-1}}^{a_{k}}\left|\frac{\partial u_{1}}{\partial x_{1}}(z)+1\right| \mathrm{d} L^{1} z & =\frac{h}{2}\left(\left|\frac{\partial u_{1}}{\partial x_{1}}\left(t_{k, j}^{(1)}\right)+1\right|+\left|\frac{\partial u_{1}}{\partial x_{1}}\left(t_{k, j}^{(2)}\right)+1\right|\right) .
\end{aligned}
$$

Thus

$$
\begin{aligned}
\sum_{k \in \tilde{R}} \int_{S_{k, j}} d(D u(x), K) \mathrm{d} L^{2} x & \geq \sum_{k \in \tilde{R}} \int_{D_{k, j}} d(D u(x), K) \mathrm{d} L^{2} x \\
& \geq \sum_{k \in \tilde{R}} \frac{h^{2}}{4}\left|\frac{\partial u_{1}}{\partial x_{1}}\left(t_{k, j}^{(1)}\right)+1\right|+\frac{h^{2}}{4}\left|\frac{\partial u_{1}}{\partial x_{1}}\left(t_{k, j}^{(2)}\right)+1\right| \\
& =\sum_{k \in \tilde{R}} \frac{h}{2} \int_{a_{k-1}}^{a_{k}}\left|\frac{\partial u_{1}}{\partial x_{1}}(z)+1\right| \mathrm{d} L^{1} z \\
& \geq \frac{11 h^{\frac{4}{3}}}{1000}
\end{aligned}
$$

contradicting the fact that the minirow $R_{i, j}$ belongs to $J_{j}$ and proving our claim.

Note that by a similar reasoning we can get

$$
\operatorname{card}(L) \leq \operatorname{card}(M)+\frac{n_{0}}{5} .
$$


Since $\operatorname{card}(R) \leq \frac{n_{0}}{500}$ it follows

$$
\begin{aligned}
\operatorname{card}(L) & =n_{0}-\operatorname{card}(M)-\operatorname{card}(R) \\
& \geq n_{0}-\operatorname{card}(L)-\frac{n_{0}}{5}-\frac{n_{0}}{500}
\end{aligned}
$$

so $2 \cdot \operatorname{card}(L) \geq \frac{500 n_{0}}{500}-\frac{100 n_{0}}{500}-\frac{n_{0}}{500}=\frac{399 n_{0}}{500}$ and hence

$$
\operatorname{card}(L) \geq \frac{15 n_{0}}{40} \text { and } \operatorname{card}(M) \geq \frac{15 n_{0}}{40} .
$$

Step 6. In this step we utilize some properties of touching squares and set up certain refinements from which we argue the final step.

Note that since none of the matrices in the set $\{U, L, R\}$ have rank one connections on the edges of our equilateral diamonds $D_{i, j}$, so for any couple of neighboring squares $S_{i, j}$ and $S_{i+1, j}$ which are both in $G$ there holds either

$$
S_{i, j} \in P_{1} \text { and } S_{i+1, j} \in P_{1}
$$

or

$$
S_{i, j} \in D_{1} \text { and } S_{i+1, j} \in D_{1} .
$$

The above means that for any "good" square we can build up a vertical interval of only "good" squares going up within $P_{1}$ or $Q_{1}$ until we hit a "bad" square. Also, given an $i \in \Phi_{1}$ and $k \in V_{i}$ since the minirow $R_{k, i}$ is at least distance $h^{\frac{2}{3}}$ away from any other minirow indexed by $V_{i}$, we thus can build up an interval of $\left[h^{-\frac{1}{3}}\right]$ squares going up (and starting from a "good" square in $R_{k, i}$ ) without crashing into any other minirow of $V_{i}$.

Let $\widetilde{G} \subset G$ be the set of squares $S_{i, j}$ for which we can find an unbroken line of $\left[h^{\frac{1}{3}}\right]$ "good" squares in $G$ vertically above and including $S_{i, j}$. Let $U_{i} \subset V_{i}$ be the subset of minirows of $R_{k, i}$ which contain at least $\frac{65 n_{0}}{80}$ squares in $\widetilde{G}$.

Define

$$
\Phi_{2}=\left\{i \in \Phi_{1}: \operatorname{card}\left(U_{i}\right) \geq \frac{\operatorname{card}\left(V_{i}\right)}{2}\right\}
$$

Now for $i \in \Phi_{2} \backslash \Phi_{1}$ we have card $\left(U_{i}\right)<\frac{\operatorname{card}\left(V_{i}\right)}{2}$ and hence for any $j \in V_{i} \backslash U_{i}$ there are at least $\frac{15 n_{0}}{80}$ "bad" squares, within $\left[h^{-\frac{1}{3}}\right]$ squares starting from some square in $R_{k, i}$. Since the minirows indexed by $\Phi_{2} \backslash \Phi_{1}$ are more than $\left[h^{-\frac{1}{3}}\right]$ squares apart, we conclude that there are at least card $\left(V_{i} \backslash U_{i}\right)$ groups of at least $\frac{15 n_{0}}{80}$ "bad" squares, that is at least

$$
\frac{\operatorname{card}\left(V_{i}\right)}{2} \cdot \frac{15 n_{0}}{80}
$$

"bad" squares in the block $B_{i}$. In view of $\operatorname{card}\left(V_{i}\right) \geq \frac{2}{30 h^{\frac{2}{3}}}$,

$$
\begin{aligned}
\operatorname{card}\left(\Phi_{2} \backslash \Phi_{1}\right) \frac{1}{30 h^{\frac{2}{3}}} \frac{15}{160 h^{\frac{2}{3}}} & \leq \operatorname{card}\left(\Phi_{2} \backslash \Phi_{1}\right) \frac{1}{30 h^{\frac{2}{3}}} \frac{15 n_{0}}{80} \\
& \leq \frac{1}{5000 h^{\frac{5}{3}}}
\end{aligned}
$$

so

$$
\operatorname{card}\left(\Phi_{2} \backslash \Phi_{1}\right) \leq \frac{320}{1000 h^{\frac{1}{3}}}
$$




$$
\operatorname{card}\left(\Phi_{2}\right) \geq \frac{9}{10 h^{\frac{1}{3}}}-\frac{32}{100 h^{\frac{1}{3}}} \geq \frac{5}{10 h^{\frac{1}{3}}} .
$$

On the other hand, for every $i \in \Phi_{2}$ there are at least $\frac{1}{30 h^{\frac{2}{3}}}$ minirows indexed by $U_{i}$. Thus for each $k \in U_{i}$ there holds

$$
P_{1} \cap R_{k, i} \geq \frac{15 n_{0}}{40} \text { and } D_{1} \cap R_{k, i} \geq \frac{15 n_{0}}{40},
$$

and by definition of $U_{i}$ we can find $\frac{15 n_{0}}{80}$ squares in $P_{1} \cap R_{k, i}$ which launch intervals of squares in $P_{1}$ of length $\left[h^{-\frac{1}{3}}\right]$ going up. In a similar fashion we find $\frac{15 n_{0}}{80}$ squares in $D_{1} \cap R_{k, i}$ which launch intervals of squares in $D_{1}$ of length $\left[h^{-\frac{1}{3}}\right]$ going up. Note that the intervals launched from squares in $R_{k, i}$ will be disjoint from those launched from $R_{l, i}$ for any $k \neq l \in U_{i}$.

Step 7. In this step we use the particular properties of the coordinate function $u_{2}$. Because $u$ is piecewise affine on the triangles $T_{i, j}^{(1)}, T_{i, j}^{(2)}$ we know that for any $S_{i, j} \in G$

$$
\sup _{z \in D_{i, j}}\left|\frac{\partial u_{2}}{\partial x_{1}}(z)\right|<\frac{1}{25}
$$

Also, when $S_{i, j} \in P_{1}$ then

$$
\sup _{z \in D_{i, j}}\left|\frac{\partial u_{2}}{\partial x_{2}}(z)\right|<\frac{1}{25}
$$

and when $S_{i, j} \in D_{1}$ then

$$
\sup _{z \in D_{i, j}} d\left(\frac{\partial u_{2}}{\partial x_{2}}(z),\{-1,1\}\right)<\frac{1}{25}
$$

Fix an $i \in \Phi_{2}$. For any $k \in U_{i}$ we can distinguish two groups of $\frac{15 n_{0}}{80}$ squares that launch intervals of length $\left[h^{-\frac{1}{3}}\right]$, contained in $P_{1}$ and $D_{1}$ respectively, all starting from the minirow $R_{k, i}$. On every interval in $P_{1}$, the function $u_{2}$ must remain within $\frac{h^{\frac{2}{3}}}{25}$ distance from a constant. On the other hand along any interval in $D_{1}$, the derivative $\frac{\partial u_{2}}{\partial x_{2}}(z)$ cannot change its sign because all the squares along this interval are "good". Thus the difference in value at one of the the endpoints of the interval must be at least $\left(1-\frac{1}{25}\right) h^{\frac{2}{3}}$. We can hence find some horizontal line $l$ of length at least $\frac{h^{\frac{2}{3}}}{4}$ such that for each $x \in l$

$$
\int_{x}^{x+o_{x} e_{1}} \frac{\partial u_{2}}{\partial x_{1}}(z) \mathrm{d} L^{1} z \geq \frac{h^{\frac{2}{3}}}{10}
$$

with some $o_{x} \in\left(0, h^{\frac{2}{3}}\right)$.

Let $\Psi_{i, k}$ denote the rectangle whose base is the line $l$ and whose height equals $h^{\frac{2}{3}}$. We have

$$
\begin{aligned}
\int_{\Psi_{i, k}} d(D u(x), K) \mathrm{d} L^{2} x & \geq \int_{x \in l} \int_{x}^{x+o_{x} e_{1}} \frac{\partial u_{2}}{\partial x_{1}}(z) \mathrm{d} L^{1} z \\
& \geq \frac{h^{\frac{4}{3}}}{40} .
\end{aligned}
$$


For every $i \in \Phi_{2}, \operatorname{card}\left(U_{i}\right) \geq \frac{\operatorname{card}\left(V_{i}\right)}{2} \geq \frac{1}{30 h^{\frac{2}{3}}}$ so letting

$$
Y=\left\{(i, j): i \in \Phi_{2}, k \in U_{i}\right\}
$$

we have

$$
\begin{aligned}
\frac{h^{\frac{1}{3}}}{500000} & \geq \sum_{(i, j) \in Y} \int_{\Psi_{i, k}} d(D u(x), K) \mathrm{d} L^{2} x \\
& \geq \operatorname{card}(Y) \frac{h^{\frac{4}{3}}}{40},
\end{aligned}
$$

and $\operatorname{card}(Y) \geq \operatorname{card}\left(\Phi_{2}\right) \frac{1}{30 h^{\frac{2}{3}}} \geq \frac{1}{60 h}$ in view of $\operatorname{card}\left(\Phi_{2}\right) \geq \frac{1}{2 h^{\frac{1}{3}}}$.

Finally we receive

$$
\frac{1}{60 h} \leq \operatorname{card}(Y) \leq \frac{40}{500000 h}
$$

which is a contradiction implied by assuming (1). The proof is done.

\section{AN APPLICATION}

As we have mentioned in the Introduction, due to the very strong result of Šverák [10] it is known that any matrix $F$ in the quasi-convex hull of the set $K=S O(2) \cup S O(2) H$ actually stays in the second lamination convex hull of these wells. From this result it could be expected that minimising the functional $(i . e$. $I(u)=$ $\left.\int_{\Omega} d(D u(x), K) \mathrm{d} L^{2} x\right)$ over the class of functions that are piecewise affine on a triangular grid and satisfy affine boundary condition $F$, the energy scales like $h^{\frac{1}{3}}$ (where $h$ is the size of the triangular mesh). Suppose we were not aware of Šverák's characterization and wanted to find it by making numerical tests. Recall that the quasiconvex hull of $K$ coincides with the set of matrices $F$ for which the two well functional can be minimised to zero over the function class with affine boundary condition $F$ (see [8], Th. 4.10). Hence if we minimise the functional over the function class $A_{h}$, we expect the energy to go to zero as we decrease the triangulation size $h$ if and only if $F$ is in the quasi-convex hull of the wells. Now due to Šverák's result, for any grid size $h$ the minimal of the functional is necessarily bounded by $C h^{\frac{1}{3}}$. On the other hand if the conjectured lower bound for the energy is true then we automatically know that numerical tests will not be able to improve on this; as we move the grid size $h$ up and down within the admissible ranges, we will see the energy of the functional always staying of the order $h^{\frac{1}{3}}$. From this we could reasonably conjecture that the quasi-convex hull of $S O(2) \cup S O(2) H$ is the second lamination convex hull (if we didn't already know it).

Let $\phi$ be now the functional having wells

$$
K=S O(3) A_{1} \cup S O(3) A_{2} \cup S O(3) A_{3},
$$

where the $A_{i}$ are given by

$$
A_{1}=\operatorname{diag}\left(\frac{1}{\lambda^{2}}, \lambda, \lambda\right), \quad A_{2}=\operatorname{diag}\left(\lambda_{1}, \frac{1}{\lambda^{2}}, \lambda\right), \quad A_{3}=\operatorname{diag}\left(\lambda_{1}, \lambda_{1}, \frac{1}{\lambda^{2}}\right)
$$

Recall the $A_{i}$ are exactly the zero energy gradients of the cubic to tetragonal phase transition. The quasi-convex hull of $K$ is unknown. However if (as it might be expected) the quasi-convex hull is equal to the $m$-th order laminate of $K$ for some $m$, then by the arguments sketched above we can conjecture that the energy of the functional when minimised over the class of piecewise affine on a triangular grid functions to scale in a very 
specific way as we move the grid size $h$ up and down. (The edges of the triangles in the mesh should not be in the set of rank one connection of $\left\{S O(3) A_{1}, S O(3) A_{2}, S O(3) A_{3}\right\}$.)

Conversely if the energy of the functional over the considered function class has the mentioned scaling, it would suggest a characterization of the quasi-convex hull of $K$. For the sake of example, since it is known [5] that $I d \in K^{(4)}$ (the 4-th lamination convex hull of $K$ ) we could take the guess that $K^{(4)}$ consists of the entire quasi-convex hull. In this case by estimating the energy of the functional acting on a fourth order laminate, the functional could be expected to scale like $h^{\frac{1}{9}}$.

Since for even the biggest computers, the range of $h$ for which we can carry out these tests is quite small, in order to observe the above mentioned scaling, it is important to have some reasonable numerical constant for $c$ in the lower bound. In this note an attempt to estimate $c$ has been made; sharper estimates would require more careful reasoning.

Acknowledgements. Georg Dolzmann read a preliminary version of this note and made some excellent suggestions for improvements. Marta Lewicka and Stefan Müller greatly improved the introduction and general presentation.

\section{REFERENCES}

[1] J.M. Ball and R.D. James, Fine phase mixtures as minimizers of energy. Arch. Rational Mech. Anal. 100 (1987) 13-52.

[2] J.M. Ball and R.D. James, Proposed experimental tests of a theory of fine microstructure and the two well problem. Philos. Trans. Roy. Soc. London Ser. A 338 (1992) 389-450.

[3] M. Chipot, The appearance of microstructures in problems with incompatible wells and their numerical approach. Numer. Math. 83 (1999) 325-352.

[4] M. Chipot and D. Kinderlehrer, Equilibrium configurations of crystals. Arch. Rational Mech. Anal. 103 (1988) $237-277$.

[5] G. Dolzmann, Personal communication.

[6] M. Luskin, On the computation of crystalline microstructure. Acta Numer. 5 (1996) 191-257.

[7] M. Chipot and S. Müller, Sharp energy estimates for finite element approximations of non-convex problems. Variations of domain and free-boundary problems in solid mechanics, in Solid Mech. Appl. 66, P. Argoul, M. Fremond and Q.S. Nguyen, Eds., Paris (1997) 317-325; Kluwer Acad. Publ., Dordrecht (1999).

[8] Variational models for microstructure and phase transitions. MPI Lecture Note 2 (1998). Also available at: www.mis.mpg.de/cgi-bin/lecturenotes.pl

[9] P. Mattila, Geometry of Sets and Measures in Euclidean Spaces, in Cambridge Studies in Advanced Mathematics, Cambridge (1995).

[10] V. Šverák, On the problem of two wells. Microstructure and phase transitions. IMA J. Appl. Math. 54, D. Kinderlehrer, R.D. James, M. Luskin and J. Ericksen, Eds., Springer, Berlin (1993) 183-189.

To access this journal online:

www.edpsciences.org 